\title{
Editorial: Biogeochemistry of Anthropogenic Particles
}

\author{
Denise M. Mitrano ${ }^{1 *}$, Antonia Praetorius ${ }^{2}$, Gaëtane Lespes ${ }^{3}$ and Vera I. Slaveykova ${ }^{4}$ \\ ${ }^{1}$ Department of Environmental Systems Science, ETH Zurich, Zurich, Switzerland, ${ }^{2}$ Department of Ecosystem \& Landscape \\ Dynamics, Institute for Biodiversity and Ecosystem Dynamics, University of Amsterdam, Amsterdam, Netherlands, \\ ${ }^{3}$ Universite de Pau et des Pays de l'Adour, E2S UPPA, CNRS, IPREM, UMR 5254, Helioparc, Pau, France, ${ }^{4}$ Environmental \\ Biogeochemistry and Ecotoxicology, Department F.-A. Forel for Environmental and Aquatic Sciences, School of Earth and \\ Environmental Sciences, Faculty of Science, University of Geneva, Genève, Switzerland
}

Keywords: nanoplastic, microplastic, nanoparticle, water, fate, transport, anthropogenic, ECOTOX

Editorial on the Research Topic

Biogeochemistry of Anthropogenic Particles

\section{INTRODUCTION}

OPEN ACCESS

Edited by:

Fereidoun Rezanezhad,

University of Waterloo, Canada

Reviewed by:

Adrian Mellage,

University of Tubingen, Germany

${ }^{*}$ Correspondence:

Denise M. Mitrano

denise.mitrano@usys.ethz.ch

Specialty section:

This article was submitted to

Biogeochemical Dynamics,

a section of the journal

Frontiers in Environmental Science

Received: 12 February 2021

Accepted: 25 February 2021

Published: 23 March 2021

Citation:

Mitrano DM, Praetorius A, Lespes G and Slaveykova VI (2021) Editorial: Biogeochemistry of Anthropogenic

Front. Environ. Sci. 9:667140. doi: 10.3389/fenvs.2021.667140
The collection of articles published in the Research Topic Biogeochemistry of Anthropogenic Particles in Frontiers in Environmental Science has provided a comprehensive perspective on the biogeochemical cycle of anthropogenic particles in the environment with an emphasis on nano- and microscale materials. The term anthropogenic particles covers a wide range of materials, including those which are manufactured or generated incidentally, or from the degradation of synthetic materials, and subsequently disseminated in(to) the environment. The aims of this Research Topic were thus to explore the state of knowledge, including major advances and challenges, with regards to the sources, occurrence, transformations, and transport of particles, as well as the interactive effects between particles and their environment including living systems. Therefore, we have brought together research papers and reviews on anthropogenic particles, regardless of their origin and nature. This integrative approach has helped to draw parallels between the knowledge already acquired in the field of anthropogenic colloids, not limited to specific chemistry or source of particles, and thus to bridge these different investigation axes to fertilize the research area dedicated to particulate contaminants. Our summary groups the articles published in the Research Topic on four levels; (1) analytical methodologies to assess particles in complex matrices, (2) connecting research across particle chemistries and sources, (3) connecting fate and transport pathways across environmental compartments and (4) connecting research across ecological impacts.

The increased usage and unprecedented release rates of anthropogenic particles into the environment, made from a plethora of materials and originating from various sources, is a specific feature of the Anthropocene. Both the short- and long-term behavior and fate of these particles will depend on their interplay with different environmental matrices over time. Here the particulate nature results in unique properties and behaviors, often not directly comparable to that of other synthetic chemicals and materials. Therefore, understanding the biogeochemistry of man-made particles remains one of the major challenges of modern environmental sciences, even after decades of study. Additionally, it is important to put these anthropogenic materials in context with natural particles, 


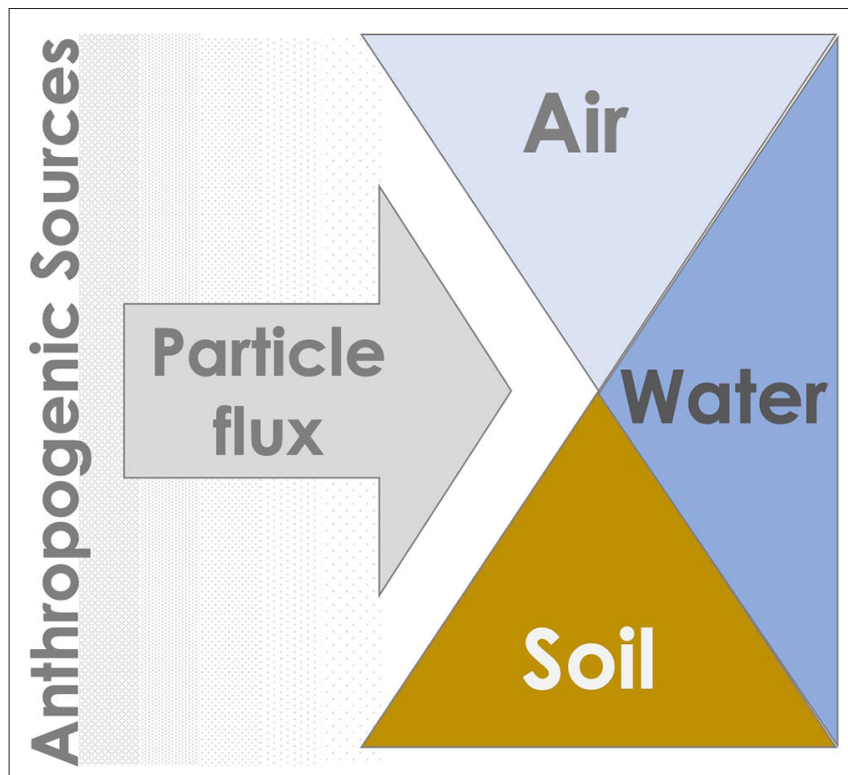

FIGURE 1 | Schematic representation of the emissions of anthropogenic particles, which can be emitted to various environmental compartments.

whose presence is often dominant in many environmental systems (Lespes et al.). Frequently, natural particles and anthropogenic particles of different origins are considered separately despite many overlapping tools, techniques, and processes, which is useful to understand behavior and interactions across different particle types. In this Research Topic, we have brought together an integrated, broad, and cross-sectoral perspective to assess particles of different origins to shed light on common underlying factors to assess the biogeochemistry of anthropogenic particles (Figure 1).

Accelerated industrialization and advances in technology have had wide reaching effects, producing particles which can range from incidental by-products of production and combustion processes (e.g., soot, street dust), to those which are intentionally produced in specific sizes and shapes for targeted applications (e.g., $\mathrm{TiO}_{2}$ in cosmetics, nano-fertilizers) or generated by the breakdown of larger products over time (e.g., microplastics). Released either intentionally or unintentionally, their occurrence in the environment is continuous, although assessing the risks of this diverse group of materials is often cumbersome as scientists grapple with (i) differentiating natural proxies from engineered counterparts, (ii) balancing positive benefits with potential environmental harm (including safer-by-design principles), and (iii) assessing which, if any, mitigation strategies would prove useful and effective. Regardless of their origin, particles can play a special role in environmental systems and behave fundamentally different in terms of fate, transport, and biological interactions than dissolved chemicals or bulk materials. Therefore, understanding the biogeochemistry of these smallest of man-made materials is crucial in assessing all the ways in which humanity is continuously impacting our environment.

\section{ANALYTICAL METHODOLOGIES TO ASSESS PARTICLES IN COMPLEX MATRICES}

Understanding the biogeochemistry of anthropogenic particles [including nanoparticles (NPs) and their aggregates] requires advanced analytical methods. Nanometrology tools have continued to improve in the last several years developing increasingly performant and standardized materials and methods. Consequently, researchers can now track particles at very low particle concentrations and tackle research projects to understand the behavior of particles in a more environmentally relevant context. Nevertheless, implementing these methods in increasingly complex real-world scenarios remains analytically challenging, and the frontier of environmental nanometrology continues to be pushed forward. This is addressed in this Research Topic through three fundamental questions:

- How can researchers detect particles in very low concentrations in complex matrices? One strategy is preconcentration of particles. This approach was investigated for suspensions of nanoplastics by Hildebrandt et al.. Very sensitive and sufficiently selective detectors can also be used, namely inductively coupled plasma-atomic spectrometry (ICP-MS). In single particle (sp) mode, ICP-MS offers particle size distributions. Triple quad MS vs. simple quad enables size-based detection limits and selectivity to be improved, as illustrated by Wojcieszek et al. with the fate of $\mathrm{TiO}_{2} \mathrm{NPs}$ investigated in a model plant.

- How can we identify the origins of nanoparticles? For the analysis of inorganic particles, ICP-MS-based approaches remain powerful. In particular when considering particle composition and speciation, using multiple analytical techniques can be advantageous. This was approached in different ways in three studies in this Research Topic: (i) sp-ICP-MS (quadrupole)/imaging/X-ray spectroscopy; (ii) sp-ICP-MS (magnetic sector)/sp-ICP-MS(time-of-flight) to identify and determine the origins and behavior of $\mathrm{CeO}_{2} \mathrm{NPs}$ and $\mathrm{TiO}_{2}$ NPs in natural waters in technical environments (Phalyvong et al.; Azimzada et al.); (iii) sample pre-fractionation/sp-ICP-MS(time-of-flight) to determine the speciation of $\mathrm{Zn}$ in colloidal phases of urban waters (Bevers et al.).

- How can we validate analytical methods? This question remains a challenge due to the lack of certified reference materials or standard suspensions for many nanomaterials and aggregates, especially in the case of plastics. In this context, labeled nanoplastics (e.g., with an incorporated metaldopant) can be a solution, as they can be more easily traced. Their value is illustrated by the validation of the method of preconcentration of nanoplastics using metaldoped polymeric NPs (Hildebrandt et al.). 


\section{CONNECTING RESEARCH ACROSS PARTICLE CHEMISTRIES AND SOURCES}

Research on the occurrence of particles and colloids in the natural environment has been conducted for many decades and is a principal component in the field of geochemistry. Some of these traditional principles and concepts may also be useful in the context of understanding the behavior of anthropogenic particles, but differences in particle material properties and chemistry changes how and what should be assessed. In this Research Topic, both engineered and incidental particles were investigated.

Improving industrial ecology is one decisive way in which the production of man-made materials can have fewer impacts at the start of a product life cycle. Slomberg et al. investigated the release of $\mathrm{TiO}_{2} \mathrm{NPs}$ from a production site, measuring industrial and urban wastewater treatment plant effluents and assessing how released particles distributed into receiving waters. The majority of the particles analyzed were confirmed to be anthropogenic, but they settled rapidly near the emission source and accumulated in the sediment. Environmental safety of engineered nanomaterials is a critical component of a "safer-by-design" approach, since we have the opportunity to control the material design and use of these materials, thereby selecting chemical properties and use scenarios which would have fewer negative environmental consequences. For the case of sunscreens, Labille et al. assessed a variety of UV-filters to review how material choice could minimize the environmental risk when particles are released into the environment during normal product use. Collectively, this information may provide industry and regulators with additional guidance on which materials should be used or avoided. Using advanced analytical tools to assess outdoor stains and paints, Azimzada et al. quantified and characterized $\mathrm{TiO}_{2}$ particles which are released during weathering processes. Here the authors describe how incorporation of $\mathrm{TiO}_{2} \mathrm{NPs}$ in different products can result in different release profiles (amounts, aggregation states) and how seasonal differences in weathering changes particle release.

In some cases, the point sources of engineered NPs are not always clear, but the particles are nevertheless detected in the environment. Phalyvong et al. correlated potential engineered NP sources with their presence in the environment, where $\mathrm{CeO}_{2}$ and $\mathrm{TiO}_{2}$ NPs were measured up and downstream of wastewater treatment plants and near a freshwater recreation center. While particles surrounding the wastewater treatment plant may have included a mixture of natural and engineered NPs, those measured near the activity center were more clearly attributed to anthropogenic sources, such as runoff from a car parking lot. While studying particle behavior directly in the environment has its advantages, the impacts of geochemistry of surface waters on particle behavior can be more systematically studied in bench-top experiments. Supiandi et al. conducted a laboratory study to assess the geochemical factors which impact the behavior CdSe/ZnS quantum dots in surface waters.

Although some nano- and microplastics (small solid polymer particles $<5 \mathrm{~mm}$ in size) are manufactured and used directly in products and applications, the vast majority of these particles in the environment originate from the abrasion of products during their lifecycle or breakdown of macroplastic litter. Halsband et al. assessed the chemical leaching profile of car tire crumb rubber to understand the potential impacts on coastal marine systems. While the leachate differed depending on tire composition and exposure scenario, organic components leached in the timespan of days while metals could still be released from the material over the 30-day test period.

Research on metals cycling processes in natural waters and their catchments has often focused on analyzing the "dissolved" fraction, which operationally is defined as the aqueous phase that passes through a $<0.45 \mu \mathrm{m}$ filter. Biogeochemical cycling of elements can occur in a dissolved state, but many elements may also be bound to/be incorporated in natural nanoparticles $(1-100 \mathrm{~nm})$ and fine colloids (100-450 nm). Thanh-Nho et al. characterized the dynamics of trace metals in a tidal creek to assess their cycling in mangroves where complex biogeochemical processes of dissolution of metal bearing mineral phases resulted in high trace metal concentrations in pore waters. Under certain conditions, elements were exported to the estuary in particulate forms even though initially the elements $(\mathrm{Fe}, \mathrm{Mn}, \mathrm{Co}$, and $\mathrm{Ni}$ ) were put into the system in dissolved forms. This gives further evidence that identifying metal state and complexation is necessary to better understand the cycling and transport of elements in catchments and to determine their fate, transport, and biological availability.

\section{CONNECTING FATE AND TRANSPORT MECHANISMS ACROSS ENVIRONMENTAL COMPARTMENTS}

The transport of particles in environmental systems is driven by a combination of their intrinsic physico-chemical properties and their extrinsic properties which result from the interplay of particles and the conditions of the surrounding medium [e.g., in terms of $\mathrm{pH}$, concentrations of various ions, presence and types of natural organic matter (NOM) or larger natural colloids]. The importance of studying particle fate as a function of a given environmental system to assess exposure and potential risks has been highlighted in several contributions to this Research Topic. Different freshwater systems have been investigated in field studies for the presence and transport of $\mathrm{Ce}-$, $\mathrm{Ti}$-, and $\mathrm{Zn}$ containing NPs (Phalyvong et al.; Slomberg et al.; Phalyvong et al.), typically with several sampling points along a given river or watershed, sometimes covering different seasons and including the sediment compartment (Slomberg et al.). The effects of varying water chemistry on NP stability and the possible formation of heteroaggregates with larger natural mineral phases are discussed by Phalyvong et al. Slomberg et al. found that most anthropogenic Ti-bearing particles occurred as aggregates in the water column, which explains the rapid sedimentation of a large fraction of the NPs close to the suspected emission sources. Additionally, they observed differences in aquatic concentrations in contrasting seasons, which may be attributed to differences in water chemistry. 
Laboratory-based studies make it possible to more systematically discern effects of water chemistry on particle properties and behavior. Supiandi et al. used synthetic river water mimicking a natural river to study the effects of specific water constituents (i.e., fulvic acid and goethite) on the speciation of CdSe/ZnS Quantum Dots (QDs). They found that the presence of fulvic acid and goethite suppressed dissolution of $\mathrm{Cd}$ and $\mathrm{Zn}$ from the QDs, making them less bioavailable to sensitive organisms. Bour et al. used artificial seawater (salinity 30\%o) and artificial freshwater (salinity 0\%o) for studying interactions of plastic microbeads and microplastic fibers with fish, amongst other aquatic organisms, but found no significant difference in exposure for the fish in the different water types.

While freshwater systems are often considered one of the first entry points for anthropogenic particles into the water cycle, the importance of better understanding the behavior and impacts of anthropogenic particles in marine environments has been highlighted multiple times in this Research Topic (Halsband et al.; Corsi et al.; Gondikas et al.). A review on the behavior and fate of representative engineered metal oxide NPs (here $\mathrm{TiO}_{2} \mathrm{NPs}$ ) and nanoplastics (here nano polystyrene) in artificial and real seawater is presented by Corsi et al. In particular, they highlight the importance of the possible formation of biomolecular coronas on the particles' surface and resulting changes in the physico-chemical properties of the NPs, as well as on their uptake by and interaction with organisms. In fact, Gondikas et al. demonstrate in their study that the presence of extracellular polymeric substances (EPS) resulting from seasonal biological activities such as algal bloom can lead to the intermittent stabilization of NPs in the marine water column. As a result, marine waters should not only be seen as a rapid sink for particles (due to the high salinity promoting aggregation and settling), but extended exposure times and further transport of NPs in the water column are possible in seasons of high biological activity. This, together with many other contributions to this Research Topic, highlights the importance of accounting for the complexity of possible processes affecting fate and sinks of anthropogenic particles in natural environments and to perform spatially and seasonally explicit fate (and risk) assessments.

\section{CONNECTING RESEARCH ACROSS ECOLOGICAL IMPACTS}

This Research Topic provides innovative insights and illustrates the large array of ongoing research on the biological assessment of the environmental impact of nano- and micro-sized anthropogenic particles that spans from aquatic (Halsband et al.; Bour et al.; Corsi et al.) to terrestrial environments (Wojcieszek et al.; Schöpfer et al.; Fringer et al.), from single-celled organisms (Fringer et al.) to biofilm communities (Desmau et al.), from plants (Wojcieszek et al.), to invertebrates and vertebrates (Bour et al.; Schöpfer et al.), from discrete nanoparticles (Wojcieszek et al.; Bour et al.; Corsi et al.; Schöpfer et al.; Fringer et al.; Desmau et al.) to more complex nanomaterials such as sunscreens (Labille et al.) or car tire crumb rubber leached chemical cocktails (Halsband et al.).
Interaction of NPs with biofilm microbial communities and consequences for NPs fate and impacts in the environment, as well as the need for further investigations were thoroughly discussed by Desmau et al.. The importance of the biofilm driven transformations such as dissolution, through ligand- or redoxmediated pathways, passivation or stabilization processes, and corona formation by EPS has the potential to strongly modify NP speciation and effects. Furthermore, biofilm-mediated NP synthesis in man-impacted systems were shown for the specific case of ZnS NPs. Using a gram-negative bacterium living in both anaerobic and aerobic environments, Fringer et al. showed that nano-polystyrene (nano-PS) were trapped by the EPS, associated to bacterial membranes and induced a specific and significant decrease in the secretion of riboflavin when exposed to nanoplastics without affecting cell viability, although the dosages used here are higher than expected for environmental concentrations nano-PS.

Wojcieszek et al. explored the uptake, translocation, and potential transformations of $\mathrm{TiO}_{2}$ NPs with different sizes in hydroponically cultivated rooted radish. Small size NPs were measured in both leaves and roots, proving the plant's ability to translocate $\mathrm{TiO}_{2}$ NPs. However, the root to shoot NP uptake ratio was low. Since NPs remained predominantly in the root, which is the edible portion of this plant, dietary exposure remains a possibility.

From a broader perspective, Corsi et al. provided an overview of the literature concerning the interaction of anthropogenic NPs with a range of marine microorganisms (from planktonic species to invertebrates and fish) and discussed the existing gaps toward a more realistic risk assessment of NPs in marine environment. $\mathrm{TiO}_{2}$, and polystyrene (nano-PS), were used as case studies to illustrate the basic concept underlying the adsorption and absorption of NPs. Modifying factors enabling a better assessment of particle uptake and related cellular pathways leading to toxic effects were considered. Interactions of the anthropogenic NPs with various naturally occurring compounds was also highlighted by Lespes et al., as well as the need of a broader and integrated approach toward fate and impact of nano-sized particles in the environment.

Finally, a comprehensive review on the exposure and hazard of nanoparticulate UV-filters $\left(\mathrm{TiO}_{2}\right.$ or $\left.\mathrm{ZnO}\right)$ of sunscreen products through the product lifecycle (manufacturing, usage, and disposal) in different compartments encountered was presented by Labille et al.. The importance of the development of realistic scenarios of environmental release and fate, as well as considering sunscreen filters and formulations which are used commercially was highlighted together with knowledge gaps. Collectively, this provides a scientific base for ecological risk assessment and toward safer by design products and sustainable nanotechnology.

Exposure to and ingestion of nano- and microplastics can impact the health, function and viability of organisms, both large and small, in a diversity of ecosystems. Halsband et al. presents the impacts of nanoplastic on a model bacterium and showed that while overall viability did not decrease, there were significant changes to cellular function, as evidenced by reduced riboflavin secretion. This impact was nanoplastic specific, since a similar finding was not observed when the bacteria were 
exposed to colloidal $\mathrm{TiO}_{2}$ or microplastic. Schöpfer et al. demonstrated that conventional and biodegradable microplastic particles were ingested by nematode, a key member of the soil food web, and induced sub-lethal effects. The alteration of the reproduction was confirmed even though other vitality metrics, such as body length, were not impacted (Thanh-Nho et al.). The observations were attributed to the physical and indirect nutritional effects rather than to chemical effects. Using a combination of microplastic morphologies (particles, fibers), feeding strategies (microplastic in presence and absence of food) and exposure conditions (fresh and salt waters), Phalyvong et al. assessed particle ingestion by fish. They found that gills were especially susceptible to microplastic exposure, and that ingestion and retention in the gut differed between particle morphologies. In many cases, the reason why microplastics are hazardous is not the polymer itself, but rather the leaching of plasticizers, additives and other compounds from the plastic over time. In addition, these same authors found that the ingestion and retention of polyethylene microbeads and doped dyed polyester microfibers differed in brine shrimp and fish (Bour et al.). Shrimps ingested large quantities of microbeads but very few microfibers, while fish ingested more microfibers than microbeads. These results suggested that particle selection can be based on size and shape, which has important implications for trophic transfer to predators.

Not only the particulate materials itself, but also different additives that can be leached from it can have significant effects on environmental biota as demonstrated by Halsband et al.. They provided a study assessing the impact of chemical additive leachates from crumb rubber granulate (CRG) produced from end of life tires exposing marine copepods. A doseresponse relationship was found with smaller lipid-poor Acartia, which showed higher morality to leachates, opposed to the larger lipid-rich Calanus, indicating species-specific differences. Benzothiazole and $\mathrm{Zn}$ were two prevailing components in the leachates. Comparing relative impacts of organisms to particles can help to elucidate if effects are observed as a specific reaction to one material (i.e., microplastic) or if organisms are simply responding to the presence of particulate matter in their environment. Consequently, adverse effects may be observed when test organisms are subjected to turbid environments regardless of the source of the particulate matter; be it from a natural or anthropogenic source. Gorokhova et al. addressed this issue by exposing the unicellular alga Raphidocelis subcapitata to both natural particles (kaolin, cellulose) and microplastics, including in combination, to assess particle property specific effects. At lower doses (10-100 mg/L), no adverse effects were observed and in fact growth stimulation was seen for natural particles compared to the control, though at very high concentrations $(1,000 \mathrm{mg} / \mathrm{L})$, organisms suffered growth inhibition from all materials. Therefore, there was less dependence on the material of the particles the organisms were exposed to, but rather the total concentration.

\section{OUTLOOK}

The contributions to the Research Topic Biogeochemistry of Anthropogenic Particles in Frontiers in Environmental Science demonstrate the broad range of sources, particle types, environments, and species, which can play a role when assessing the fate and possible impacts of man-made particles to the natural environment. While ultimately particle chemistry will play an important role in biogeochemical of particle fluxes in the environment, researchers who study particle behavior of one class of materials can significantly advance their own studies by understanding the analytical toolboxes and concepts used by researchers who study different material types. Many parallels can be drawn between engineered, incidental and natural particles, as well as between different material types, such as metal/metal oxide NPs or nano- and microplastics. Fate, transport and (adverse) effects are impacted by particles size and shape, as well as by the interactions at the interface between particle surface and environment. These interactions are influenced by particle chemistry and by the conditions of the surrounding environment. Understanding and predicting these complex interactions will be instrumental in assessing potential risks posed by anthropogenic particles in the environment and help us design effective mitigation measures as well as more benign alternatives.

\section{AUTHOR CONTRIBUTIONS}

All authors collectively wrote and edited this editorial.

Conflict of Interest: The authors declare that the research was conducted in the absence of any commercial or financial relationships that could be construed as a potential conflict of interest.

Copyright (C) 2021 Mitrano, Praetorius, Lespes and Slaveykova. This is an open-access article distributed under the terms of the Creative Commons Attribution License (CC $B Y)$. The use, distribution or reproduction in other forums is permitted, provided the original author(s) and the copyright owner(s) are credited and that the original publication in this journal is cited, in accordance with accepted academic practice. No use, distribution or reproduction is permitted which does not comply with these terms. 